\title{
Steatohepatitic Variant of Hepatocellular Carcinoma: A Focused Review
}

\author{
Andrea Morgan Olofson ${ }^{\mathrm{a}}$, David Hernandez Gonzalo ${ }^{\mathrm{a}}$, \\ Michael Chang, ${ }^{\mathrm{a}, \mathrm{b}}$, Xiuli Liu ${ }^{\mathrm{a}, \mathrm{c}}$
}

\begin{abstract}
The incidence of hepatocellular carcinoma (HCC) has steadily increased over the past three decades and currently ranks as the fifth most common cancer worldwide. Likewise, non-alcoholic fatty liver disease (NAFLD), a known risk factor for the development of HCC, has emerged as the most common liver disease in Western countries. The underlying pathogenesis of NAFLD-related HCC remains unclear. The steatohepatitic variant of $\mathrm{HCC}$ (SH-HCC) typically presents in patients with metabolic risk factors and either cirrhotic or non-cirrhotic NAFLD and shares many of the histological features found in non-alcoholic steatohepatitis (NASH). Given their similar morphological features, distinguishing SH-HCC from background fatty liver can be a diagnostic challenge. Immunohistochemical studies to characterize and assist in the diagnosis are relatively limited. Whether the steatotic phenotype of SH-HCC results from the tumor's adaptive response to an environment rich in fatty acids or from an independent pathogenic pathway remains to be seen. This review aims to summarize what is currently known regarding the pathogenesis and clinicopathological features of SH-HCC.
\end{abstract}

Keywords: Steatohepatitic; Hepatocellular carcinoma; NAFLD; NASH; Metabolic syndrome

\section{Introduction}

The incidence of primary liver cancers in the USA has more than tripled over the past three decades, rising from 2.6 per 100,000 in 1975 to 8.9 per 100,000 in 2015 [1]. Hepatocellular carcinoma (HCC) constitutes $70-85 \%$ of all primary liver tumors and ranks as the fifth most common cancer and the second most common cause of cancer deaths worldwide [2,

Manuscript submitted November 2, 2018, accepted November 22, 2018

aDepartment of Pathology, Immunology, and Laboratory Medicine, College of Medicine, University of Florida, Gainesville, FL, USA

bPathology and Laboratory Medicine Service, Malcom Randall VA Medical Center, Gainesville, FL, USA

${ }^{\mathrm{c} C}$ Corresponding Author: Xiuli Liu, Department of Pathology, Immunology, and Laboratory Medicine, PO Box 100275, Gainesville, FL 32610, USA. Email:xiuliliu@ufl.edu

doi: https://doi.org/10.14740/gr1110
3]. Multiple chronic liver diseases have been associated with the development of $\mathrm{HCC}$, including hepatitis $\mathrm{C}$ virus (HCV) infection, hepatitis $\mathrm{B}$ virus (HBV) infection, alcohol liver disease and non-alcoholic fatty liver disease (NAFLD). NAFLD is the most common liver disease in Western countries, with a burden of cirrhosis twice that of chronic HCV infection, and is expected to soon become the most common precipitating factor for liver transplantation [4]. Multiple mechanisms underlying obesity-driven hepatocarcinogenesis have been proposed, including elevated proinflammatory cytokines, adipokine dysregulation, oxidative and endoplasmic reticulum stress, and alterations in gut microbiota [5]. However, the pathogenesis of NAFLD-related HCC and the impact such a process has on tumor phenotype remains unclear and is likely multifactorial. The histology of steatohepatitic hepatocellular carcinoma (SH-HCC) has been described in resection specimens. Unfortunately, diagnostic criteria to aid in the evaluation of biopsy specimens have not been well established; therefore, the diagnosis of SH-HCC in biopsies remains challenging.

\section{Clinical Features}

HCC has a striking variety of histological variants including fibrolamellar, scirrhous, clear cell, biphenotypic, lymphocyte-rich, sarcomatoid, cirrhotomimetic, granulocyte colony-stimulating factor (G-CSF) producing, and the most recently described, SHHCC. In 2010, Salomao et al [6] first recognized SH-HCC in explant livers with chronic HCV infection and noted that it shared many of the morphological features of steatohepatitis, such as large droplet steatosis, ballooning malignant hepatocytes, Mallory-Denk bodies and pericellular fibrosis [6]. They reported that SH-HCC was present in $35 \%$ of HCC cases in explant livers with chronic HCV infection, and of these cases, two-thirds of the patients possessed risk factors for NAFLD [6]. Furthermore, the non-neoplastic background liver in $63 \%$ of the SH-HCC cases demonstrated NAFLD-like changes superimposed on those of chronic HCV infection. From these findings, they proposed a potential link between NAFLD in the setting of HCV infection and the subsequent development of SH-HCC [6].

In a follow-up study, Salomao et al identified the SH-HCC variant in $14 \%$ of all cases of $\mathrm{HCC}$ from liver resections and explants [7]. Each of these cases, except one, occurred in patents with steatohepatitis. SH-HCC was found more often in patients with either non-alcoholic steatohepatitis (NASH) or 

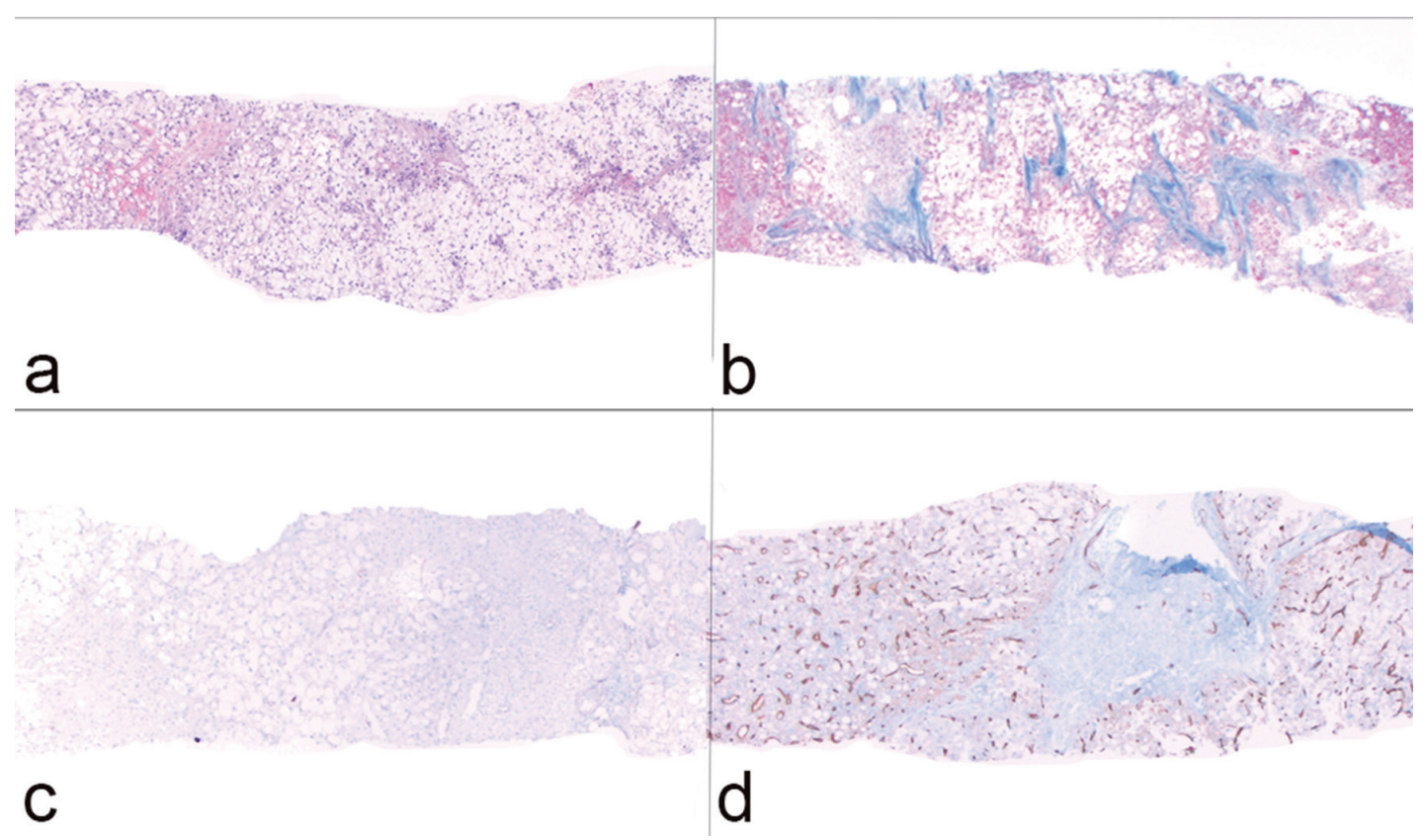

Figure 1. Histological features of steatohepatitic hepatocellular carcinoma (SH-HCC). (a) Core biopsy of hepatic parenchyma with extensive steatosis (H\&E, $\times 40)$; (b) trichrome stain highlights thick bands of fibrosis with bridging ( $\times 40)$; (c) absence of bile ducts/portal tracts as demonstrated by immunostain for cytokeratin 7 ( $\times 40)$; (d) capillarization of tumor sinusoids highlighted by immunostain for CD34 (× 40).

alcoholic steatohepatitis (ASH) compared to those with other underlying chronic liver diseases. In addition, patients with $\mathrm{SH}-\mathrm{HCC}$ were found to have a higher prevalence of metabolic syndrome risk factors when compared to patients with conventional HCC. These findings further supported a potential causative association between SH-HCC, metabolic syndrome and steatohepatitis [7]. Similar findings were recapitulated in a study undertaken by Jain et al which found that in 101 cases of HCC from explanted livers, steatohepatitic morphology was significantly associated with NAFLD and the underlying presence of metabolic risk factors [8].

In 2015, Yeh et al undertook a study to determine whether the presence of steatohepatitic features in HCC was due to: 1) tumor cells retaining the capacity to undergo morphological changes secondary to underlying metabolic factors or 2) genetic features inherent to SH-HCC tumor cells that result in a steatohepatitic phenotype [9]. They identified and described 12 cases of SH-HCC arising in patients without metabolic syndrome or fatty liver disease, suggesting that in a subset of cases, the steatohepatitic morphology results from tumorspecific genetic alterations. HCC is known to be a genetically heterogeneous entity, and little is yet known about the specific mutational drivers underlying SH-HCC. Therefore, these findings present an intriguing question as to the existence of common etiological factors that may be found independently or concurrently in NAFLD and SH-HCC.

\section{Pathologic Features of Resected SH-HCC}

Macroscopically, resected SH-HCC tends to be slightly firmer and more golden-yellow in color compared to other $\mathrm{HCC}$ variants due to the relatively greater degree of fibrosis and steatosis [7]. Tumors are nodular, well-demarcated and range in size from 0.5 to $11 \mathrm{~cm}$ [6-10]. SH-HCC is defined by the presence of features typical of steatohepatitis including large-droplet steatosis, fibrosis, ballooning of malignant hepatocytes, Mallory-Denk bodies and inflammation. These features may be present in varying combinations; however, they should constitute $\geq 5 \%$ of the tumor volume. SH-HCC often exhibits an infiltrative growth pattern into nearby portal tracts. Mitotic activity is minimal. The fibrosis pattern of $\mathrm{SH}-\mathrm{HCC}$ can include a trabecular pattern with thick, irregular bands of fibrous tissue with a haphazard distribution or a pericellular "chicken-wire" pattern with thin, delicate fibrous bands interweaving between tumor cells [6]. As in other variants of HCC, the absence of portal tracts in the lesion remains a key feature (Figs. 1, 2).

\section{Immunohistochemistry}

Distinguishing SH-HCC from background NAFLD can be challenging due to its infiltrative as opposed to expanding growth pattern, minimal mitotic activity and variable pleomorphism. Immunohistochemical studies to characterize SH-HCC are relatively limited. Jain et al found that 13 out of $18 \mathrm{SH}-$ HCC cases demonstrated glypican-3 positivity [8]. Similarly, Salomao et al identified glypican-3, glutamine synthetase and heat shock protein-70 (HSP-70) positivity in 10 of $10 \mathrm{SH}-\mathrm{HCC}$ cases [6]. However, these markers may not be sensitive. For example, in a study evaluating 70 cases of SH-HCC, diffuse 


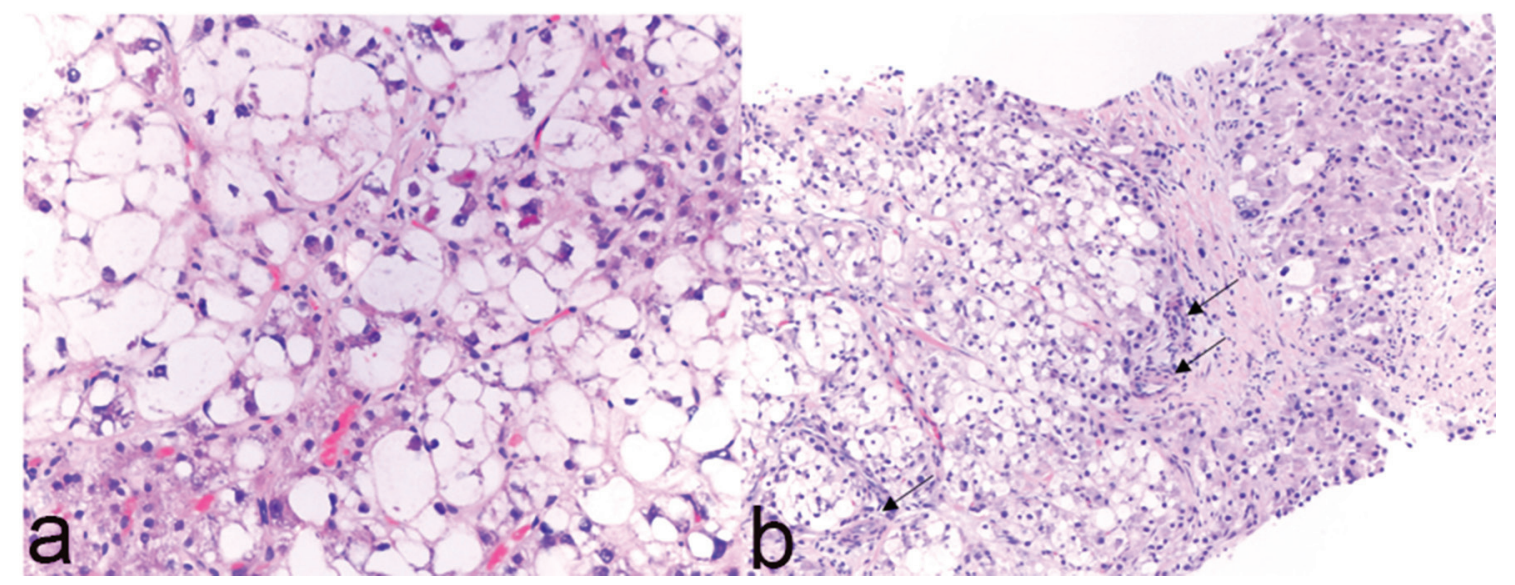

Figure 2. Histological features of steatohepatitic hepatocellular carcinoma (SH-HCC). (a) High magnification $(\mathrm{H} \& \mathrm{E}, \times 400)$ reveals steatosis, ballooning and Mallory-Denk bodies within tumor cells; (b) isolated arterioles (arrows) without associated portal tracts $(H \& E, \times 200)$.

and intense staining with glutamine synthetase was found to be much less common in SH-HCC occurring in $4 \%$ of cases compared to $26 \%$ of conventional HCC cases [3]. Likewise, nuclear $\beta$-catenin staining was present in $6 \%$ of SH-HCC cases versus $25 \%$ of conventional HCC cases [3]. However, sonic hedgehog ligand expression was present in $90 \%$ of SH-HCC tumors and $83 \%$ of conventional HCC tumors [3].

\section{Pathology of SH-HCC on Biopsy Material}

The diagnosis of SH-HCC on biopsy material can be extremely challenging. First, SH-HCC is a newly-described entity with limited published data to date addressing the histological and immunohistochemical features that can assist in its diagnosis. Second, these tumors commonly occur in a background of steatohepatitis, which shares multiple overlapping features with SH-HCC, including minimal to mild pleomorphism. In this setting, a reticulin stain is not particularly helpful in distinguishing benign fatty liver parenchyma from SH-HCC due to the presence of intratumor pericellular fibrosis which can complicate the assessment of liver plate thickness, and the loss of reticulin staining that can occur in benign fatty liver tissue [11]. As previously discussed, invasion into nearby portal tracts by SH-HCC is a useful histological feature on resection specimens; however, this feature may not be present on limited biopsy specimens. Although isolated arterioles are often used as histological clues for the diagnosis of hepatocellular neoplasms, morphologically similar central arterialization can be seen in steatohepatitis [12]. Studies focusing on the more subtle histomorphological features of SH-HCC as well as the genetic alterations and protein expression profiles of SH-HCC are urgently needed. For now, one must take extra care in using the features of steatohepatitis-like changes, widened plates and/or rosettes/pseudoglands to make a diagnosis of SH-HCC on limited biopsy material [13]. A diagnosis of SH-HCC on biopsy may be made based on a combination of features such as absence of portal tracts, isolated arterioles, widened trabecular plates and capillarization of sinusoids (Figs. 1,2).

\section{Differential Diagnosis}

Another diagnostic pitfall includes the distinction of SH-HCC from steatohepatitis-like changes in focal nodular hyperplasia (FNH). The classic morphological features associated with FNH include nodular architecture, thick fibrous septa, abnormal vessels and ductular reaction. Steatosis can be seen in approximately half of all cases and Mallory-Denk bodies in approximately $20 \%$ [14]. In one recent study characterizing 33 cases of FNH, steatohepatitic features, including ballooning hepatocytes and Mallory-Denk bodies, were identified in 54\% of cases [13]. To help distinguish between these two entities, the authors make note that the presence of ductular reaction in fibrous septa lacking native bile ducts was present in all cases of steatotic FNH, as opposed to SH-HCC, which does not demonstrate ductular reaction [13]. Unfortunately, the pattern of fibrosis may be of less utility. Although FNH typically demonstrates thick fibrous bands from which thinner septa may radiate, it has been known to demonstrate a pericellular pattern. Conversely, SH-HCC typically demonstrates a pericellular pattern; however, thick trabecular fibrous bands may also be present. Another important diagnostic clue is the presence of thick-walled vessels within the dense fibrous septa of FNH. This feature is not found in SH-HCC [13].

Table 1 summarizes the histological and immunohistochemical features of SH-HCC, steatohepatitis, conventional HCC and FNH with steatohepatitic features with data mostly generated from resected specimens.

\section{Oncogenesis}

One recently proposed explanation for the steatotic phenotype of SH-HCC and its association with fatty liver disease may lie in how cancer cells selectively evolve to survive in a lipid-rich environment as in patients with NAFLD. Selective metabolic reprogramming of cancer cells to adapt to local environmental conditions is a hallmark of carcinogenesis. Although fatty acid 
Table 1. Comparison of SH-HCC, Steatohepatitis, Conventional HCC and Steatohepatitic FNH on Resection

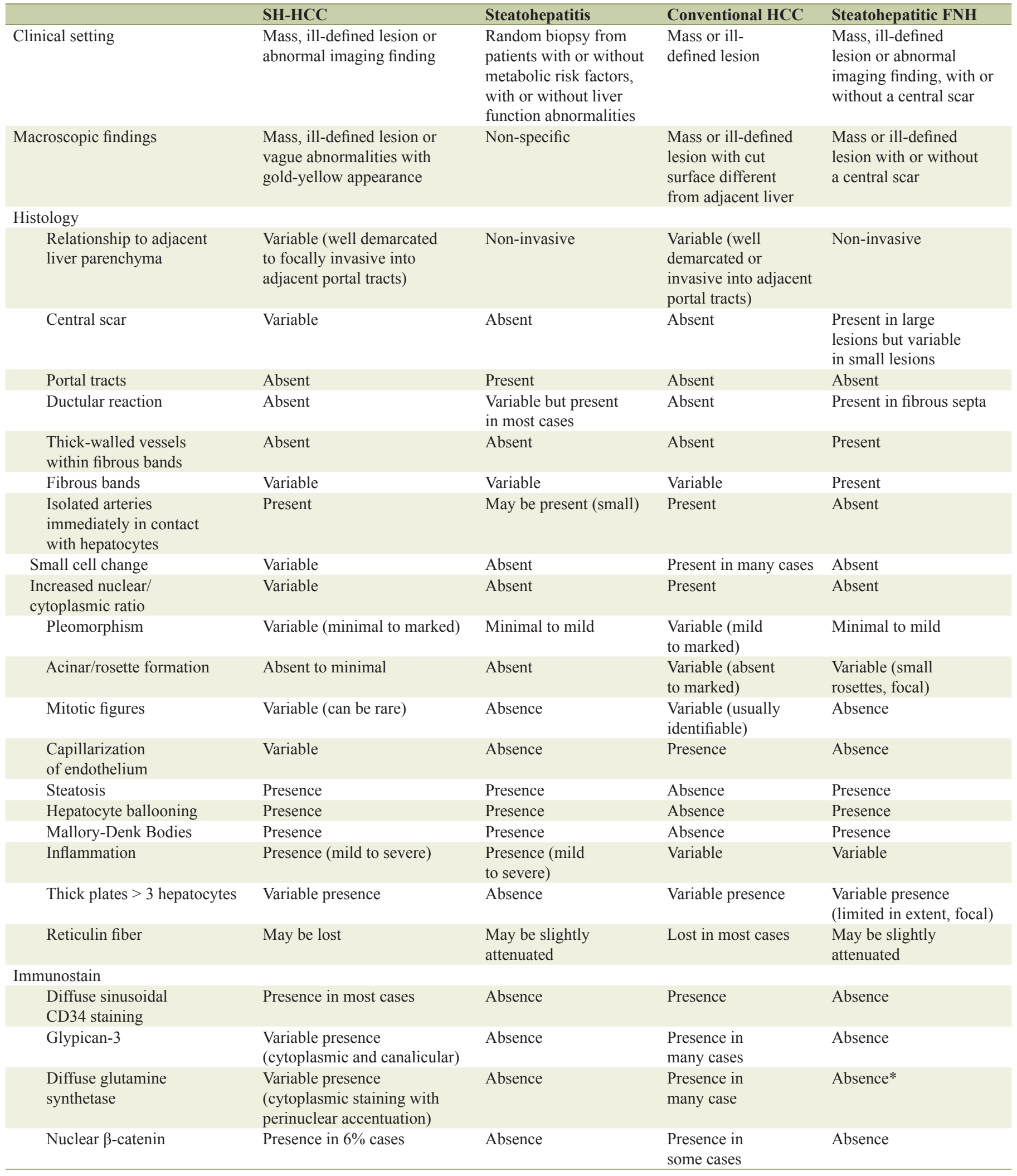

SH-HCC: steatohepatitic hepatocellular carcinoma; HCC: hepatocellular carcinoma; FNH: focal nodular hyperplasia. *It usually shows a map-like staining pattern. 


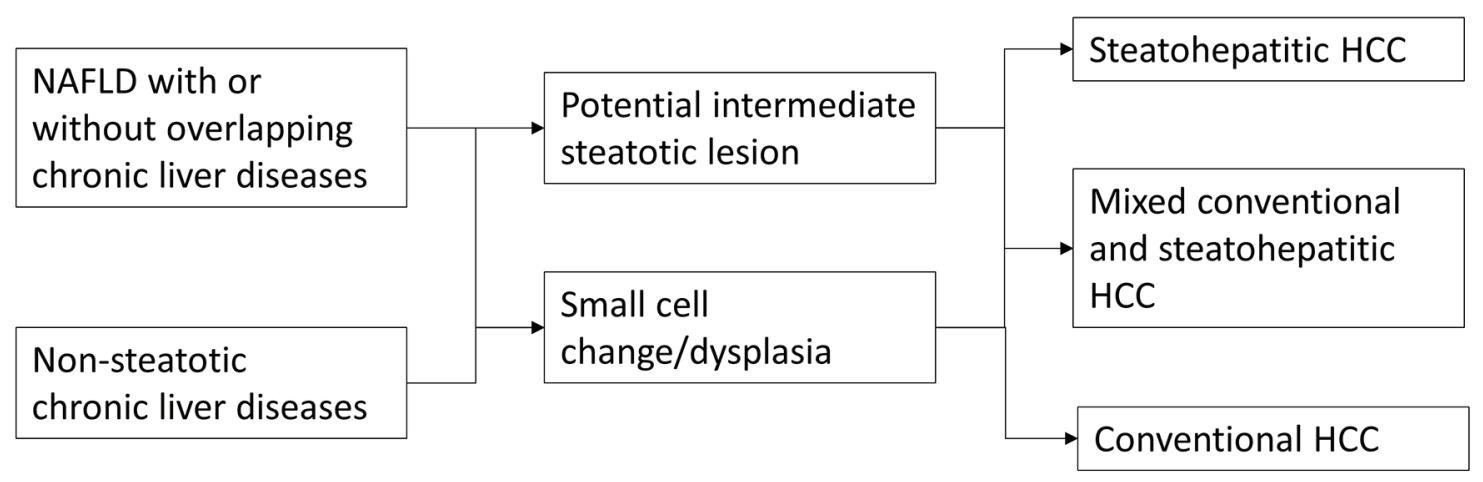

Figure 3. Potential tumorigenic pathways in the development of steatohepatitic hepatocellular carcinoma.

oxidation is often used by cancer cells to generate energy, excessive fatty acid oxidation due to high intracellular fat levels can lead to metabolic stress and eventually lipotoxic cell death, e.g. ballooning degeneration [5]. In patients with NASH, extremely high levels of fatty acids from dietary intake and lipolysis of visceral adipose tissue can lead to lipotoxic hepatocyte death. This lipotoxic effect is an important promoter of NASH-associated $\mathrm{HCC}$, and thus, HCC must adapt in order to survive in such a lipid-rich environment. In mouse models, the downregulation of carnitine palmitoyltransferase 2 (CPT2), the rate-limiting enzyme of fatty acid oxidation, has been associated with lipotoxicity-resistant HCC cells and the accumulation of acylcarnitine [5, 15]. The resulting excessive accumulation of acylcarnitine was found to enhance STAT3 activation, thereby promoting hepatocarcinogenesis via the IL-6/STAT3 pathway [5].

Alternatively, SH-HCC arising in patients with non-steatotic chronic liver diseases may acquire steatohepatitic features secondarily to an inflammation-induced metaplastic phenomenon rather than an adaptation to high levels of fatty acids. A molecular study undertaken by Calderaro et al demonstrated that although the morphologic features of SH-HCC suggest dysregulation of metabolic pathways, no significant mutations were identified in genes associated with fatty acid synthesis, glycogenesis or neoglucogenesis [16]. However, an association was identified between the SH-HCC phenotype and activation of the IL6/JAK/STAT3 pathway and C-reactive protein (CRP) immunohistochemical expression [16].

One interesting and important question that remains to be determined is the existence of a potential intermediate lesion or step that lies between non-neoplastic liver and SH-HCC such as a steatohepatitic adenoma or steatohepatitic dysplasia. One recent report suggested an association between inflammatory hepatocellular adenoma and sonic hedgehog hepatocellular adenoma with higher body mass index (BMI) [17]. Additional research is needed to answer this clinically and conceptually important question. A simple diagram outlining potential steps in the tumorigenesis of SH-HCC in NAFLD and other chronic liver diseases is illustrated in Figure 3.

\section{Survival}

So far, a limited number of reports have suggested that there is no statistically significant difference in overall survival, disease-free survival, development of metastatic disease or local recurrence between patients with $\mathrm{SH}-\mathrm{HCC}$ versus those with conventional HCCs $[7,18]$. However, one study demonstrated that patients with SH-HCC tend to have better disease-free survival [19].

\section{Conclusions}

As the incidence and prevalence of obesity and metabolic syndrome continue to rise worldwide, NAFLD has replaced viral disease and alcohol abuse as the main driver for the development of HCC [2]. Given its association with NAFLD, obesity and metabolic syndrome, it is reasonable to expect the incidence of SH-HCC to follow suit. This is of particular concern given the difficulties encountered in the histological diagnosis of SH-HCC in the background of steatohepatitis, particularly in biopsy material. With continued investigation, a better understanding of the pathogenic pathways underpinning the development of SH-HCC will become crucial for the development of diagnostic tools and the identification of therapeutic targets for this growing patient population.

\section{Conflict of Interest}

None.

\section{Financial Support}

None.

\section{References}

1. Noone AM, Howlader N, Krapcho M, Miller D, Brest A, Yu M, Ruhl J, et al (eds). SEER Cancer Statistics Review, 1975-2015, National Cancer Institute. Bethesda, MD, https://seer.cancer.gov/csr/1975_2015/, based on November 2017 SEER data submission, posted to the SEER web site, April 2018. 
2. Marengo A, Rosso C, Bugianesi E. Liver cancer: connections with obesity, fatty liver, and cirrhosis. Annu Rev Med. 2016;67:103-117.

3. Ando S, Shibahara J, Hayashi A, Fukayama M. betacatenin alteration is rare in hepatocellular carcinoma with steatohepatitic features: immunohistochemical and mutational study. Virchows Arch. 2015;467(5):535-542.

4. Chalasani N, Younossi Z, Lavine JE, Charlton M, Cusi $\mathrm{K}$, Rinella M, Harrison SA, et al. The diagnosis and management of nonalcoholic fatty liver disease: Practice guidance from the American Association for the Study of Liver Diseases. Hepatology. 2018;67(1):328-357.

5. Fujiwara N, Nakagawa H, Enooku K, Kudo Y, Hayata Y, Nakatsuka T, Tanaka Y, et al. CPT2 downregulation adapts HCC to lipid-rich environment and promotes carcinogenesis via acylcarnitine accumulation in obesity. Gut. 2018;67(8):1493-1504.

6. Salomao M, Yu WM, Brown RS, Jr., Emond JC, Lefkowitch JH. Steatohepatitic hepatocellular carcinoma (SHHCC): a distinctive histological variant of HCC in hepatitis C virus-related cirrhosis with associated NAFLD/ NASH. Am J Surg Pathol. 2010;34(11):1630-1636.

7. Salomao M, Remotti H, Vaughan R, Siegel AB, Lefkowitch JH, Moreira RK. The steatohepatitic variant of hepatocellular carcinoma and its association with underlying steatohepatitis. Hum Pathol. 2012;43(5):737-746.

8. Jain D, Nayak NC, Kumaran V, Saigal S. Steatohepatitic hepatocellular carcinoma, a morphologic indicator of associated metabolic risk factors: a study from India. Arch Pathol Lab Med. 2013;137(7):961-966.

9. Yeh MM, Liu Y, Torbenson M. Steatohepatitic variant of hepatocellular carcinoma in the absence of metabolic syndrome or background steatosis: a clinical, pathological, and genetic study. Hum Pathol. 2015;46(11):1769-1775.

10. Jain D, Nayak NC, Saigal S. Hepatocellular carcinoma in nonalcoholic fatty liver cirrhosis and alcoholic cirrhosis: risk factor analysis in liver transplant recipients. Eur J Gastroenterol Hepatol. 2012;24(7):840-848.
11. Singhi AD, Jain D, Kakar S, Wu TT, Yeh MM, Torbenson M. Reticulin loss in benign fatty liver: an important diagnostic pitfall when considering a diagnosis of hepatocellular carcinoma. Am J Surg Pathol. 2012;36(5):710-715.

12. Gill RM, Belt P, Wilson L, Bass NM, Ferrell LD. Centrizonal arteries and microvessels in nonalcoholic steatohepatitis. Am J Surg Pathol. 2011;35(9):1400-1404.

13. Deniz K, Moreira RK, Yeh MM, Ferrell LD. Steatohepatitis-like changes in focal nodular hyperplasia, a finding to distinguish from steatohepatitic variant of hepatocellular carcinoma. Am J Surg Pathol. 2017;41(2):277-281.

14. Nguyen BN, Flejou JF, Terris B, Belghiti J, Degott C. Focal nodular hyperplasia of the liver: a comprehensive pathologic study of 305 lesions and recognition of new histologic forms. Am J Surg Pathol. 1999;23(12):14411454.

15. Lin M, Lv D, Zheng Y, Wu M, Xu C, Zhang Q, Wu L. Downregulation of CPT2 promotes tumorigenesis and chemoresistance to cisplatin in hepatocellular carcinoma. Onco Targets Ther. 2018;11:3101-3110.

16. Calderaro J, Couchy G, Imbeaud S, Amaddeo G, Letouze E, Blanc JF, Laurent C, et al. Histological subtypes of hepatocellular carcinoma are related to gene mutations and molecular tumour classification. J Hepatol. 2017;67(4):727-738.

17. Nault JC, Couchy G, Balabaud C, Morcrette G, Caruso S, Blanc JF, Bacq Y, et al. Molecular classification of hepatocellular adenoma associates with risk factors, bleeding, and malignant transformation. Gastroenterology. 2017;152(4):880-894 e886.

18. Lee JS, Yoo JE, Kim H, Rhee H, Koh MJ, Nahm JH, Choi JS, et al. Tumor stroma with senescence-associated secretory phenotype in steatohepatitic hepatocellular carcinoma. PLoS One. 2017;12(3):e0171922.

19. Shibahara J, Ando S, Sakamoto Y, Kokudo N, Fukayama M. Hepatocellular carcinoma with steatohepatitic features: a clinicopathological study of Japanese patients. Histopathology. 2014;64(7):951-962. 